\title{
From Acoustic Waves to Microwaves
}

\author{
G. Mendes Pacheco ${ }^{a, *}$, J.M. Salvi Sakamoto ${ }^{b}$ And C. Kitano ${ }^{c}$ \\ ${ }^{a}$ Department of Microwave and Optoelectronics, Instituto Tecnológico de Aeronáutica, \\ 50, Praça Marechal Eduardo Gomes, São José dos Campos, São Paulo 12228-900, Brazil \\ ${ }^{b}$ Division of Photonics, Instituto de Estudos Avançados, Trevo Coronel Aviador José Alberto Albano do Amarante, \\ São José dos Campos, São Paulo 12228-001, Brazil \\ ${ }^{c}$ Department of Electric Engineering, Universidade Estadual Paulista, \\ Campus III, Ilha Solteira, São Paulo 15385-000, Brazil
}

\begin{abstract}
We present here some results of our research related to the optoelectronics and photonics and show all the experimental setups used. Starting with a discussion on the importance of the waves, we demonstrate our achievements based on employment of acoustic, optical, and microwaves and their technological use. The results concern the acousto-optic and electro-optic effects. The generalized analysis of the electro-optic effect reveals a new high induced birefringence in lithium niobate. A patented optical fiber microphone is presented, and its applications to the measurements of acoustic wave velocity in gases and in the laser ultrasound non-destructive evaluation system are discussed. Finally, the generation of microwaves by an optical method with substantial cost reduction is presented.
\end{abstract}

DOI: $10.12693 /$ APhysPolA.127.25

PACS: 43.35.Sx, 42.79.Jq, 51.40.+p, 42.79.-e, 43.35.Zc, 84.40.-x, 85.60.- $\mathrm{q}$

\section{Introduction}

The word "wave" is so meaningful for those tracking the nature and related achievements from the scientific and technological point of view. The wave comprehension, theoretically and experimentally, changed so much along the history. The acoustic and electromagnetic waves are directly related to sensors, featuring mixing effects, mixer devices, and modulations. In this paper, some results of the research related to the acoustic waves, optical waves, and microwaves are discussed, with a special emphasis on the optically generated signals. All this work has been done in Brazil. The topic of the research belonged to the field of optoelectronics, and more recently, to the field of photonics. The subjects of research presented here can be summarized as follows: acousto-optic and electrooptic effects, acousto-optical spectrometer, optical microphone, laser ultrasound, optical microwave oscillator.

\section{Acousto-optic and electro-optic effects}

Starting with the acousto-optic and electro-optic effects, the characteristics of acoustic and optical waves are related to the material properties of their propagation media. In our laboratory, the bulk optical modulators based on quartz, lithium niobate, and tellurium dioxide were constructed with an operation frequency from $20 \mathrm{MHz}$ to $40 \mathrm{MHz}$.

The acousto-optic effect was considered in two aspects. Initially, the basics of the acousto-optic interaction itself

*corresponding author; e-mail: gpacheco@ita.br were the subject of analysis. With a better comprehension of the acousto-optic interaction acquired, the applications became the aim.

An extended work on the combination of acousto-optic and electro-optic effects was conducted in order to overcome the trade-off between the diffraction efficiency and bandwidth [1]. The phase mismatching is a key parameter for the acousto-optic interaction. The control of this important parameter by adjusting the refraction index via the electro-optic effect is the basic idea to combine those two effects. To investigate the sensibility of the refraction index to the direction of applied electric field $E$, a new generalized coordinate system was used. The new system has three orthogonal axes $X_{1}$, $X_{2}$, and $X_{3}$. The generalization of the electro-optic effect analysis comes from an arbitrary orientation of $X_{1} X_{2} X_{3}$ system (to write the index ellipsoid) with respect to the $X Y Z$ crystallographic axes. The applied electric field was considered to be always directed along the $X_{1}$ axis, and the set of the Euler angles [2] was used to point the electric field along an arbitrary direction.

Calculations of the natural and induced birefringence were performed for different cases of the optical beam and electric field directions: (1) the optical beam propagates along $X_{1}$, the longitudinal electro-optic effect, (2) the optical beam propagates along $X_{2}$ or $X_{3}$, the transverse electro-optic effect.

Lithium niobate was considered as the electro-optically active medium, the optical wavelength was equal to $0.632 \mu \mathrm{m}$ and the amplitude of the applied electric field was equal to $5000 \mathrm{~V} / \mathrm{m}$. Figure 1 shows the orientation of axes corresponding to the maximum induced birefringence, which is obtained at the angle $\psi$ equal to $41^{\circ}$ for the electric field applied along $X_{1}$ and optical propagation along $X_{2}[3]$. 


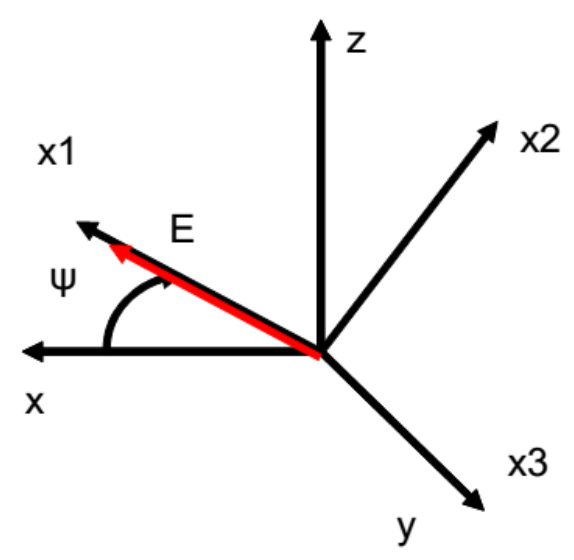

Fig. 1. Orientation of applied electric field in the coordinate system with axes $X_{1}, X_{2}, X_{3}$ rotated with respect to the crystallographic axes $X, Y, Z$. The Euler angles: $\varphi=0^{\circ}, \theta=90^{\circ}, \psi$ from $0^{\circ}$ to $90^{\circ}$; optical propagation along $X_{2}$.

\section{Acousto-optic spectrometer}

In order to practically apply the acousto-optic effect, a set of acousto-optic spectrometers was implemented. They were used at the Itapetinga Radio Observatory (ROI), where a radio astronomy spectrometers development program was conducted.

Considering the applications of the acousto-optic effect a set of acousto-optic spectrometers were constructed. The main results were obtained at the Radio Astronomy Observatory of the ITAPETINGA, ROI, where a radio astronomy spectrometers development program was conduced. The main results were in the field of galactic astronomical radio sub-millimeter sources observations [4] with a great sensibility and wide instantaneous receiver intermediate frequency bandwidth of $100 \mathrm{MHz}$ [5].

\section{Optical microphone}

\subsection{Implementation}

The detection of acoustic waves is an intense field of research with several different applications. We investigated the optical microphone based on optical fibers. The interest in this device comes from the advantages it offers as compared to conventional sensors, such as the electrical and chemical passiveness and immunity to electromagnetic interference (EMI) [6-9].

The general scheme of the optical microphone is shown in Fig. 2. The optical microphone we initially made comprised a light source, two step-index optical fibers, a lens, a membrane, and a photo-detector. The light source was a He-Ne laser with the $633 \mathrm{~nm}$ wavelength and $10 \mathrm{~mW}$ power. A dielectric mica film was used as reflective membrane. The lens had a $5 \mathrm{~mm}$ focal distance, and a PIN photodiode OPF480 was employed as photodetector. The entire optical system was mounted on a trail over a granite table. The optical microphone experimental setup is depicted in Fig. 3.

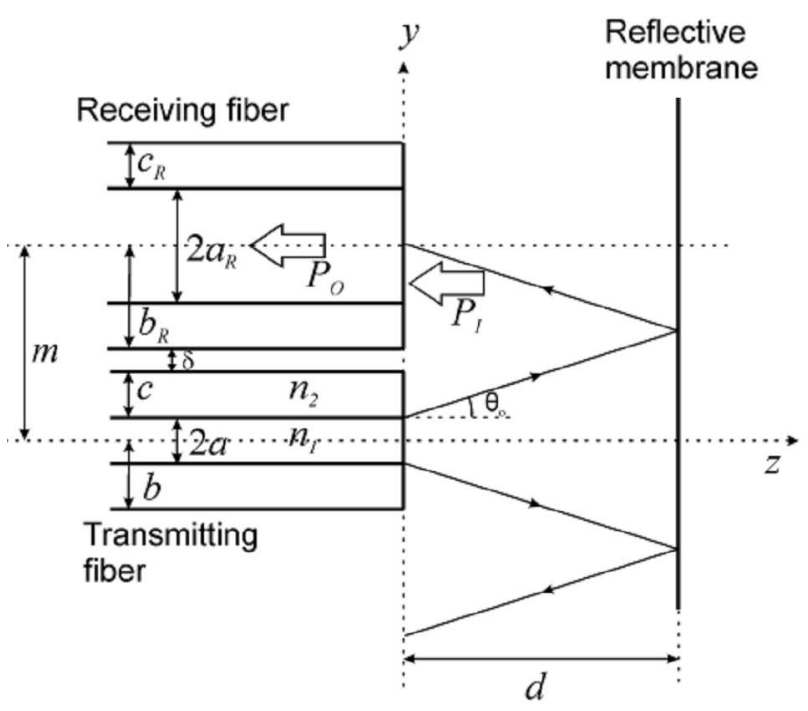

Fig. 2. General scheme of optical microphone.

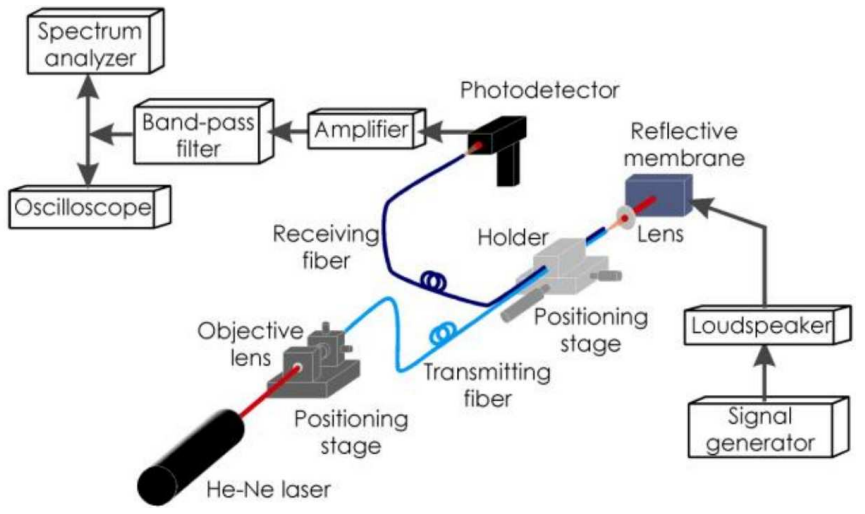

Fig. 3. Optical microphone setup.

The output spectrum of the optical microphone was analyzed with the input sinusoidal signal at frequencies 2,4 , and $6 \mathrm{kHz}$. The spectrum for each signal is shown in Fig. 4. The dynamic range measured from these spectra is better than $50 \mathrm{~dB}$ with a noise level around $-80 \mathrm{~dB}$ up to $12.5 \mathrm{kHz}$. In view of the results obtained, in addition to the low cost and simplicity of the configuration, this system becomes competitive compared to more costly and complex systems, which use DFB lasers, acousto-optical modulators and fiber optic directional coupler [6], fiber bundle [7], and interferometer mounting [10].

Two important applications of the developed optical microphone are presented in the present paper. The first one is characterization of gases by measuring the speed of sound, which is described below. Next, the laser ultrasound application for non-destructive evaluation (NDE) is described.

\subsection{Sound speed measurements}

The optical microphone was used to examine the possibility of measuring the speed of sound in gases [11]. This work was stimulated by the research program for rocket 


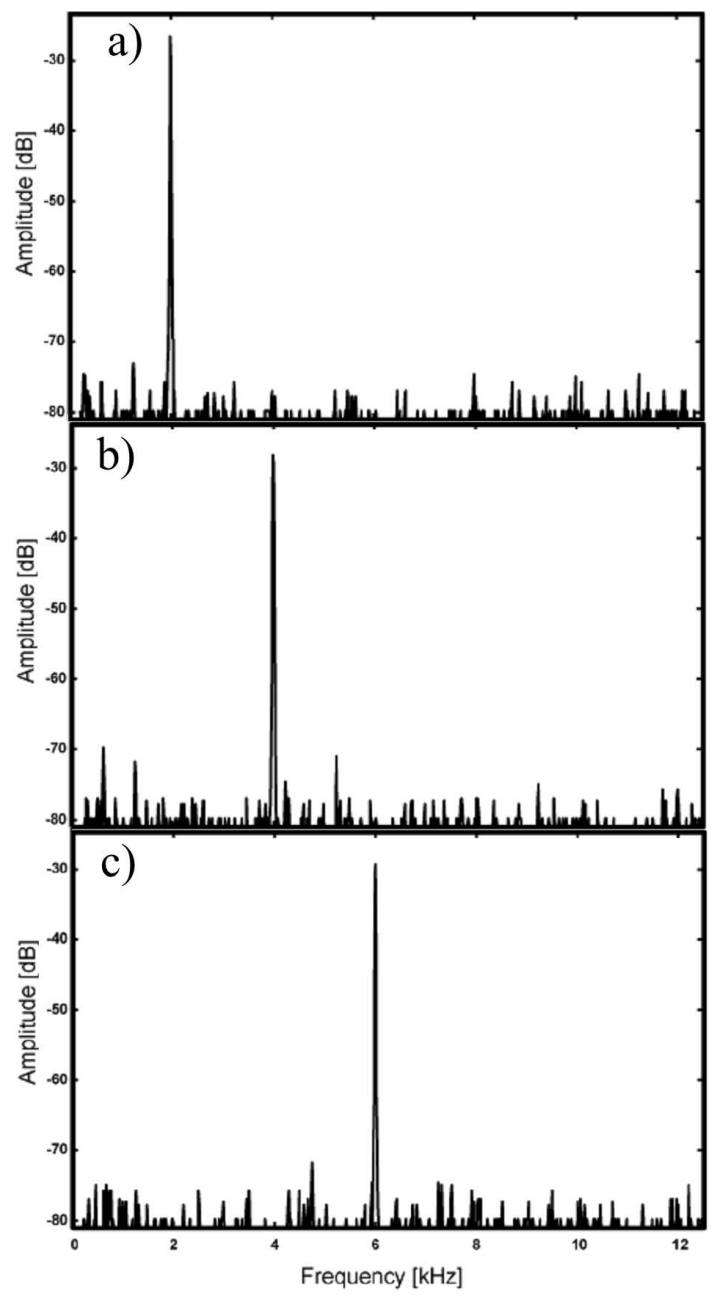

Fig. 4. Frequency domain output at (a) $2 \mathrm{kHz}$, (b) $4 \mathrm{kHz}$, (c) $6 \mathrm{kHz}$.

engine development. Measurements of the sound speed in a gas flow obtained directly from a rocket engine test ("bank shot") are of great interest since they allow for assessment of the actual performance of the engine.

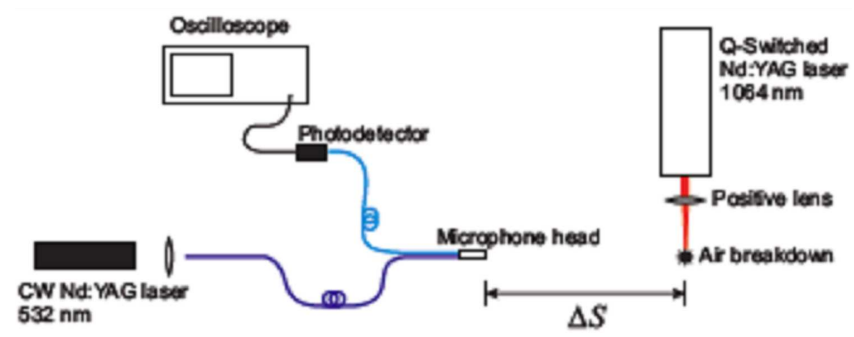

Fig. 5. Optical microphone setup for sound speed measurements.

The setup for measuring the sound speed in gases is shown in Fig. 5. It comprises the $Q$-switched Nd:YAG laser with the following characteristics: wavelength of $1064 \mathrm{~nm}$, pulse width (FWHM) of $9 \mathrm{~ns}$, maximum energy per pulse of $420 \mathrm{~mJ}$, and maximum repetition rate of $10 \mathrm{~Hz}$. The laser beam focused by the positive lens (focal length $100 \mathrm{~mm}$ ) triggers an electrical breakdown in the dielectric gas (air, in the case described here), which in turn generates an acoustic pulse. That pulse travels a distance, reaches the optical microphone and the detected signal is registered by a digital oscilloscope. The light emitted by the air breakdown acts as a trigger, detected directly by the photodetector. From the time difference between the trigger and the acoustic pulse, the sound velocity in the air is calculated.

The mean value of the obtained speed of sound is $342.3 \mathrm{~m} / \mathrm{s}$ with a standard deviation of $1.1 \mathrm{~m} / \mathrm{s}$, i.e., with precision of $0.3 \%$. These results agree very well with theoretical values [12].

\subsection{Laser ultrasound for nondestructive evaluation}

After the results with the optical microphone in gases were obtained, the system shown in Fig. 3 was applied for detection of laser generated acoustic waves in solids. In this case, the reflective membrane was replaced by the surface of a solid under test. The $Q$-switched Nd:YAG laser (the same as described above) was used to generate acoustic waves in solid samples as shown in Fig. 6. In some cases, the generation of acoustic waves occurred in the ablative regime [13]. A new sensor was constructed specially for the purpose of the laser ultrasound tests. It was initially used for characterization of a piezoelectric transducer, and later served as a part of the laser ultrasonic NDE setup.

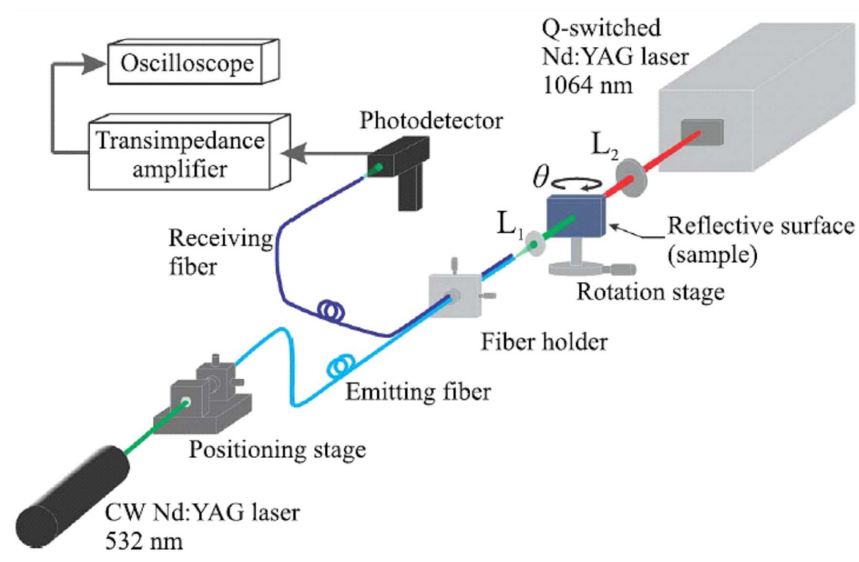

Fig. 6. Laser ultrasound setup for nondestructive evaluation.

The velocities of longitudinal, shear, and surface acoustic waves were measured in aluminum samples yielding the values of $6.43,3.17$, and $2.96 \mathrm{~mm} / \mu \mathrm{s}$, respectively, with an error smaller than $1.3 \%$ [14]. Our sensor proved to be a suitable alternative to piezoelectric or interferometric detectors for detection of ultrasonic waves, timeof-flight measurements, and nondestructive inspection.

\section{Optical oscillator for microwave signal generation}

The optoelectronic generation of microwaves by an optical oscillator was first reported during the 1990's [15]. 
The optoelectronic oscillator (OEO) is a ring optical circuit with a fiber-optic length closing the feedback path. The feedback length determines the time delay that defines the frequency step in the radio frequency output spectrum. The OEO is a dual output generator. It has one output in the optical domain, which can be used to send a microwave signal along an optical fiber link, and the other output in the radio frequency domain, which releases locally an electrical high-frequency signal.

During the OEO development, we introduced small but important changes as compared to the first OEO reported before. The delay element we used was not an optical fiber but a coaxial cable after the photodetector. The delay occurred in the radio frequency domain and not in the optical domain within the optical fiber. Evidently, using an optical fiber the same delay time $\tau$ can be obtained with less weight and volume than using a coaxial cable. However, replacing the optical fiber by the coaxial cable as a delay element is based on the two important reasons. First, the optical fiber is very sensitive to temperature, and the effects of radiation into a space are not completely understood. A coaxial cable of comparatively small length $(1 \mathrm{~m})$ provides a cheap, stable and reliable delay component that can be easily constructed. Secondly, substitution of the dual-output modulator by the single-output one leads to cost reduction.

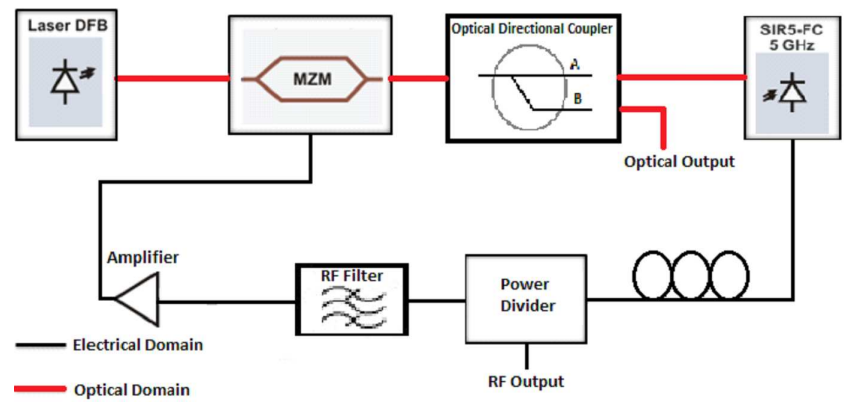

Fig. 7. Schematic of optoelectronic oscillator.

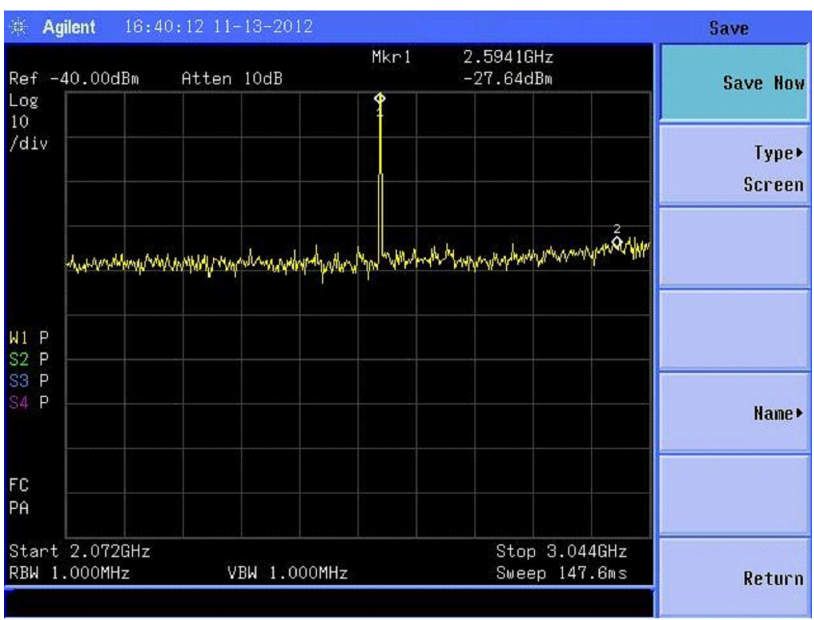

Fig. 8. Spectrum of the optoelectronic oscillator radiofrequency output signal.
The schematic of the optoelectronic oscillator constructed is shown in Fig. 7 with the optical and electric domains indicated. The amplification for the feedback loop gain (in the electrical domain) was $40 \mathrm{~dB}$. The light source was a distributed feedback (DFB) laser pigtailed with an output power up to $20 \mathrm{~mW}$ and operating at $1500 \mathrm{~nm}$. Instead of a dual-output MachZehnder (MZ) optical modulator, a single-output modulator followed by a directional coupler with output ratio equal to $30 / 70$ was used.

Figure 8 shows the $2.5 \mathrm{GHz}$ spectral component at the oscillator RF output [16]. The line width is less than $30 \mathrm{kHz}$ and the output power is $-27 \mathrm{dBm}$. Such a signal exists also in the optical domain. This allows for transmission of the signal from the optical output of the oscillator using an optical link. This is important, since the distributed radar stations are the basic systems to be connected to a remote OEO.

\section{Final comments}

A set of results dealing with acoustic waves, electromagnetic waves and microwave signals was presented. The results are related to the laser modulation, spectral analysis of microwave signals based on acousto-optic spectrometers, acoustic wave detection, nondestructive evaluation in laser ultrasound systems and the microwave generation by optical methods.

\section{References}

[1] D.H. Psaltis, H. Lee, G. Sirat, Appl. Phys. Lett. 46, 215 (1985).

[2] H. Goldstein, Classical Mechanics, Addison Wesley, New York 1950

[3] G.M. Pacheco, M.Sc. Dissertation, Instituto Tecnológico de Aeronáutica, Săo José dos Campos 1989.

[4] G.M. Pacheco, E. Scalise Jr., in: Astrophysical Maser, Eds. G. Nedoluha, A.W. Clegg, Springer, Berlin 1993.

[5] G.M. Pacheco, Ph.D. Thesis, Instituto Nacional de Pesquisas Espaciais, Săo José dos Campos 1994.

[6] J.P.F. Wooler, B. Hodder, R.I. Crickmore, Meas. Sci. Technol. 18, 884 (2007).

[7] J.A. Bucaro, N. Lagakos, B.H. Houston, J. Acoust. Soc. Am. 118, 1406 (2005).

[8] M. Feldmann, S. Büttgenbach, in: Proc. IEEE Sensors 2005, Irvine, CA 2005, p. 769.

[9] J.M.S. Sakamoto, G.M. Pacheco, Phys. Proced. 3, 651 (2010).

[10] L. Kruger, H.J. Theron, in: Int. Microwave $\&$ Optoelectronics Conf. IMOC, Bahia, 2007.

[11] J.M.S. Sakamoto, G.M. Pacheco, C. Kitano, H.A. Machado, AIP Conf. Proc. 1433, 737 (2012).

[12] J. Eargle, The Microphone Workbook, Focal Press, Burlington 2004, p. 7.

[13] J.M.S. Sakamoto, B.R. Tittmann, A. Baba, G.M. Pacheco, J. Phys. Conf. Ser. 278, 1 (2011).

[14] J.M.S. Sakamoto, C. Kitano, G.M. Pacheco, B.R. Tittmann, Appl. Opt. 51, 4841 (2012).

[15] X.S. Yao, L. Maleki, Opt. Soc. Am. 13, 1725 (1996).

[16] L.A.D. Britto, M.Sc. Dissertation, Instituto Tecnológico de Aeronáutica, São José dos Campos 2013. 